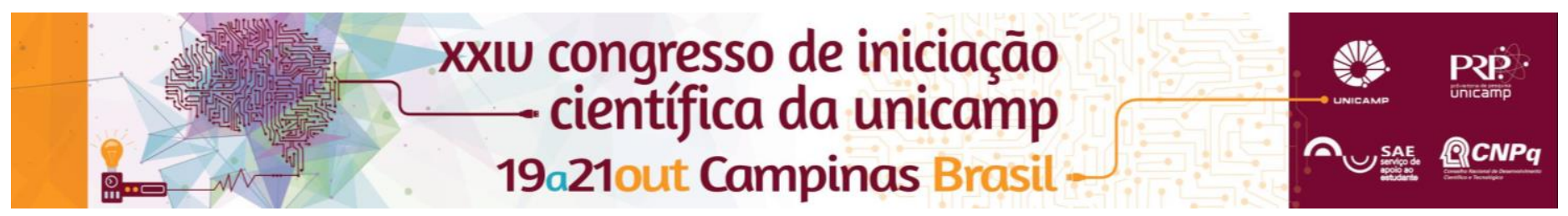

\title{
Study of the crystal quality in detwinned BaFe2As2by Xray diffraction.
}

\section{Carlos M. G. A. de Mayolo (PQ), Caio R. S. dos Santos (IC).}

\begin{abstract}
In the present work we measured the crystal quality of BaFe2As2 samples in room and low temperatures using single crystal Xray diffraction. We then proceeded to try and detwine [1] them as a way of correcting the distortions in the crystaline structure caused by the phase transition that happens in low temperatures.
\end{abstract}

\section{Key words:}

$X$-ray, Superconductors, BaFe2As

\section{Introduction}

In this work we studied BaFe2As2 single crystal samples that were grown at the GPOMS laboratory. First we characterized the sample at room tempearture, measuring it's Bragg peaks, then we brought the samples to very low temperature and again characterized it. In doing so, we can see that below the phase transition temperature some of the peaks split near it's regular angular position. We tried to find a way to remove the twinned domains that causes the splitting and loss of crystal quality.

\section{Results and Discussion}

We have measured the samples in a range of temperatures and have assured that the splitting occured. After long alignments and measurements of the orientation matrix we tried heating the sample with a laser to see if the transferred energy was enough to cause the phase transition to the tetragonal phase, in which we hadn't got much success. We then proceeded to try to obtain difratograms for the samples where the twinned peaks would not appear in the low temperature range. For this we had some special sample holders designed for this purpose.

\section{Conclusions}

The data gathered were hard to analyze due to various factors that impaired our work this year, but dispite that fact we were able to see evidences of some phenomena that happens in the crystalline structure of the compound that seems really promising for studies yet to come.

\section{Acknowledgement}

I want to acknowledge professor Dr. Carlos Giles for all the advising and the opportunity given in participating in this project and much more during this year.

1. M. A. Tanatar, E. C. Blomberg, A. Kreyssig,1 M. G. Kim, N. Ni, A. Thaler, S. L. Bud'ko, P. C. Canfield, A. I. Goldman, I. I. Mazin, and R. Prozorov, Uniaxialstrain mechanical detwinning of $\mathrm{CaFe} 2 \mathrm{As} 2$ and $\mathrm{BaFe} 2 \mathrm{As} 2$ crystals: Optical and transport study. Phys. Rev. B 81, 184508, May 2010, doi: 10.1103/PhysRevB.81.184508. 